\title{
Quality of Life Comparison between Vertebroplasty and Kyphoplasty in Patients with Osteoporotic Vertebral Fractures
}

\author{
Su-Keon Lee ${ }^{1}$, Seung-Hwan Lee ${ }^{1}$, Sang-Pil Yoon ${ }^{1}$, Young-Tae Lee ${ }^{1}$, Geun Jang ${ }^{1}$, \\ Sang-Youn Lim ${ }^{2}$, Hwan-Mo Lee ${ }^{2}$, Seong-Hwan Moon ${ }^{2}$, Kyeong-Seop Song ${ }^{1}$ \\ ${ }^{1}$ Department of Orthopaedic Surgery, Gwangmyeong Sungae Hospital, Gwangmyeong, Korea \\ ${ }^{2}$ Department of Orthopaedic Surgery, Yonsei University College of Medicine, Seoul, Korea
}

Study Design: Retrospective evaluation.

Purpose: To compare quality of life in postmenopausal women with osteoporotic vertebral fractures (OVFs) who underwent vertebroplasty (VP) or kyphoplasty (KP).

Overview of Literature: Patient with OVFs who do not respond to conservative treatment can be treated with VP or ballon KP for faster pain relief. There are controversies on which procedure is more effective.

Methods: Five hundred twenty-eight postmenopausal women in nationwide hospitals with age of 50 years or older who underwent VP of KP for OVFs were enrolled in this study. Health related quality of life was measured using the European Quality of Life 5 Domains (EQ-5D) and visual analogue scale (VAS).

Results: In the VP group, average EQ-5D dimension was 1.95 in mobility, 1.86 in self care, 2.02 in usual activity, 2.19 in pain, 1.69 in anxiety or depression. In the KP group, average EQ-5D dimension was 1.83 in mobility, 1.78 in self care, 1.98 in usual activity, 2.03 in pain, 1.55 in anxiety or depression. Quality of life of KP group was significantly better than that of the VP group in mobility $(p=0.016)$, pain $(p=0.001)$, and anxiety or depression $(p=0.008)$. Average EQ-5D index of the VP and the KP group was $0.353( \pm 0.472)$ and 0.485 $( \pm 0.357)$, respectively. The EQ-5D index of the KP group was significantly $(p<0.001)$ higher than that of the KP group. The difference of VAS between VP and KP group was not statistically significant $(p=0.580)$.

Conclusions: Quality of life in patient with OVFs who underwent KP was significantly better than that of patients who underwent VP.

Keywords: Osteoporotic spinal fracture; Vertebroplasty; Kyphoplasty; Quality of life

\section{Introduction}

Osteoporotic vertebral fractures (OVFs) occur more frequently than ankle, wrist or hip fractures. OVFs constitute major health problem to patients, resulting in worse quality of life compared to normal people [1-4].
Because of the poor quality of osteoporotic bone, classical open surgery with metal implants often fails, contributing to persistent back pain, neurological symptoms and functional limitations [5]. Vertebroplasty (VP) and balloon kyphoplasty (KP) are less invasive procedures to treat OVFs. Recently, two randomized controlled trials

Received Jul 22, 2014; Revised Aug 10, 2014; Accepted Aug 24, 2014

Corresponding author: Kyeong-Seop Song

Department of Orthopaedic Surgery, Gwangmyeong Sungae Hospital,

\#36 Digital-ro, Gwangmyeong 423-711, Korea

Tel: +82-2-2680-7699, Fax: +82-2-2680-7755, E-mail: sksub@paran.com 
have indicated that both VP and KP can produce immediate pain relief compared to conservative treatments $[6,7]$.

There are many literatures that compared the effectiveness and complications of the two procedures [812]. Comparison studies demonstrated similar results in terms of pain and functional status improved by VP or KP [13,14]. Kim et al. [15] reported that KP had a significant advantage over VP in terms of kyphosis correction, vertebral height restoration, and cement leakage prevention. However, few reports compared the quality of life after the two procedures. Therefore, the objective of this study was to compare the quality of life in postmenopausal women with OVFs who received VP or KP.

\section{Materials and Methods}

Five hundred twenty-eight postmenopausal women from 17 study sites in nationwide hospitals with age of 50 years or older who underwent VP or KP for OVFs were enrolled in this study. Enrollment was taken between April 2008 and January 2009. Total number of the VP group was 283 and that of the KP group was 245 .

\section{Inclusion and exclusion criteria}

The patients who had osteoporosis (T-score below, -2.5 ) and morphometric vertebral fracture on lateral thoracic and lumbar radiograph with one of the following criteria were included: 1) Height loss of more than $2.5 \mathrm{~cm}$ during the last 1 year; 2) Local tenderness on fracture site; 3) Evidence of recent fracture on magnetic resonance imaging or whole body bone scan; 4) History of trauma during last 3 months; 5) Surgery for compression fracture during last 3 months.

Of 528 patients with OVFs, 283 who underwent VP were assigned into the VP group, and 245 patients who underwent KP were assigned into the KP group. Indication of both procedures was persistent pain after sustained bracing and medical treatment for about two weeks. Quality of life of these patients was measured using a questionnaire that contained the European Quality of Life 5 Domains (EQ-5D) and visual analogue scale (VAS) after the two procedures. The exclusion criteria were: a history of cancer, spinal disorder, infection, or inability to communicate.

\section{Measures analyzed}

Our main outcome, quality of life, was measured by the EQ-5D and VAS. The EQ-5D is a 5-domain, 3-response option scale. On the 3-response option scale, level 1 was defined as having no problems in the EQ-5D domain, level 2 was defined as having some problems, and level 3 was defined as having severe problems in that domain. Each of the possible 243 health states was mapped to a country-specific preference-based value or utility, in which 1.00 represented full health and 0.00 represented a state equivalent to death [16]. EQ-5D index was calculated using the country specific preference-based value.

The VAS score was between 0 and 100, with 0 representing the worst health status whereas 100 representing the best health status. EQ-5D scale and VAS were reported directly by the patient. A total of 65 orthopedic physicians participated in this study.

\section{Statistical analysis}

EQ-5D dimensions and VAS were compared between the VP group and the KP group using $t$-test. Statistically significant difference was considered when $p$-value was less than 0.05 .

\section{Results}

Average ages of the VP group and the KP group were $72.58( \pm 7.95)$ years and $73.94( \pm 6.92)$ years, respectively. The difference of age between the two groups was not statistically significant $(p=0.134)$. Average bone mineral density of the VP group was $0.47( \pm 0.22) \mathrm{mg} / \mathrm{cm}^{2}$, which was significantly $(p=0.001)$ lower than that $(0.56 \pm 0.16$ $\mathrm{mg} / \mathrm{cm}^{2}$ ) of the KP group. Chronic disease properties of patients in the two groups are summarized in Table 1.

Both procedures were performed most commonly at the lumbar spine level. The thoracic spine was the second most frequently performed level (Table 2).

In the VP group, average value of each EQ-5D dimension was 1.95 in mobility, 1.86 in self care, 2.02 in usual activities, 2.19 in pain, 1.69 in anxiety or depression. In the KP group, average value of each EQ-5D dimension was 1.83 in mobility, 1.78 in self care, 1.98 in usual activities, 2.03 in pain, 1.55 in anxiety or depression. The quality of life in the VP group was significantly worse than that of the KP group in mobility $(p=0.016)$, pain 
Table 1. Descriptive data of the vertebroplasty group and the kyphoplasty group

\begin{tabular}{lccc} 
Variable & Vertebroplasty group $(\mathrm{n}=283)$ & Kyphoplasty group $(\mathrm{n}=245)$ & $p$-value \\
\hline Age $(\mathrm{yr})$ & $72.58( \pm 7.95)$ & $73.94( \pm 6.92)$ & 0.134 \\
\hline Lumbar BMD $\left(\mathrm{mg} / \mathrm{cm}^{2}\right)$ & $0.47( \pm 0.22)$ & $0.56( \pm 0.16)$ & \\
\hline Chronic disease & & & \\
\hline Hypertension & $122(43)$ & $104(42)$ \\
\hline Diabetes mellitus & $16(5)$ & $14(6)$ \\
\hline Heart disease & $16(5)$ & $9(3)$ \\
\hline Arthritis & $2(1)$ & $3(1)$ \\
\hline No chronic disease & $105(37)$ & $90(37)$ \\
\hline
\end{tabular}

Values are presented as mean \pm standard deviation or number (\%).

$\mathrm{BMD}$, bone mineral density.

Table 2. Site of osteoporotic vertebral fractures in the vertebroplasty group and the kyphoplasty group

\begin{tabular}{lcr} 
Variable & Vertebroplasty group (n=283) & Kyphoplasty group (n=245) \\
Cervical spine (\%) & $4(1)$ & $1(<1)$ \\
Thoracic spine (\%) & $123(43)$ & $111(45)$ \\
Lumbar spine (\%) & $155(55)$ & $130(53)$ \\
Sacrum (\%) & $1(<1)$ & $3(1)$ \\
\hline
\end{tabular}

Table 3. Comparison of EQ-5D and VAS between the vertebroplasty group and the kyphoplasty group using $t$-test

\begin{tabular}{|c|c|c|c|}
\hline EQ-5D dimensions & Vertebroplasty group (n=283) & Kyphoplasty group (n=245) & $p$-value \\
\hline Mobility & $1.95( \pm 0.63)$ & $1.82( \pm 0.56)$ & $0.016^{*}$ \\
\hline Self-care & $1.87( \pm 0.71)$ & $1.78( \pm 0.66)$ & 0.172 \\
\hline Usual activities & $2.02( \pm 0.65)$ & $1.98( \pm 0.49)$ & 0.368 \\
\hline Pain & $2.19( \pm 0.61)$ & $2.03( \pm 0.47)$ & $0.001^{*}$ \\
\hline Anxiety or depression & $1.69( \pm 0.66)$ & $1.55( \pm 0.60)$ & $0.008^{*}$ \\
\hline EQ-5D index & $0.353( \pm 0.472)$ & $0.485( \pm 0.357)$ & $<0.001^{*}$ \\
\hline VAS & $57.49( \pm 24.35)$ & $56.41( \pm 20.64)$ & 0.580 \\
\hline
\end{tabular}

Values are presented as average (standard deviation).

EQ-5D, European Quality of Life 5 Domains; VAS, visual analogue scale.

${ }^{*} p$-value less than 0.05 .

$(p=0.001)$, and anxiety or depression $(p=0.008)$. Average EQ-5D index in the VP group was $0.353( \pm 0.472)$, which was significantly $(p<0.001)$ lower than that $(0.485 \pm 0.357)$ of the KP group. In the VP group, average VAS was 57.49, which was not significantly $(p=0.580)$ different from that (56.40) of the KP group (Table 3 ).

\section{Discussion}

OVFs are the most frequently observed pathology and the leading cause of morbidity and mortality in geriatric patients [13]. Progressive loss of posture as a result of OVFs is one of the major problems associated with its enormous impact on the quality of life. A vertebral deformity leads to relapse of pain, cosmetic problems, decreased activity, change in mood, and reduced pulmonary function, which subsequently results in a decrease in thoracic volume and an increased risk of further fractures of the adjacent vertebra [14].

Initial therapies for OVFs are sustained bracing, pro- 
longed bed rest, and medical treatment with nonsteroidal anti-inflammatory drugs, narcotic medication and antiresorptive therapy. However, some patients fail to benefit from these treatment modalities, resulting in persistent disease-related morbidity. The pain and incapacity resulting from OVFs demand the development of new treatment modalities as effective therapy.

The following two related techniques were developed to regain vertebral body height and function as well as to decrease pain and incapacity: 1) percutaneous VP, in which cement is inserted into the vertebral body to reinforce the fractured bone; and 2) KP, a modification of VP in which a balloon is inserted and inflated in a compressed vertebral body to reestablish the height of the compressed bone [17]. Clinical studies have reported that similar results in terms of pain and functional status improvement are achieved from both VP and KP $[13,14]$. However, it is currently unclear whether VP or KP can provide better clinical outcome. Few studies have compared the quality of life between the two procedures. In our study, we compared the outcomes between VP and $\mathrm{KP}$ in treating painful OVFs with regard to the quality of life using EQ-5D and VAS. The difference of VAS between VP and KP group was not statistically significant $(p=0.580)$. However, in the aspect of EQ-5D, there were statistical differences between the two groups on mobility, pain and anxiety or depression. Our results indicated that $\mathrm{KP}$ resulted in significantly better quality of life compared to VP.

Klazen et al. [6] reported that VP resulted in greater pain relief than conservative treatment. Differences in mean VAS scores between baseline and 1 month were -5.2 and -2.7 after VP and conservative treatment, respectively. Differences in mean VAS scores between baseline and 1 year were $-5.7(-6.22$ to -4.98$)$ and $-3.7(-4.35$ to -3.05$)$ after VP and conservative treatment, respectively [6]. In our study, in each dimension of EQ-5D, pain was the most impaired dimension of quality of life. However, the quality of life after VP or KP was not compared to that at the preoperative status. Therefore, we assumed that the pain was significantly decreased after VP or KP. However, many patients suffered from persistent back pain.

Some studies have reported that KP has a significant advantage over VP in terms of kyphosis correction and vertebral height restoration $[15,18,19]$. Suzuki et al. [20] reported that initial severe deformation of OVF was the worst prognostic factor for severe lasting pain and disability, resulting in the deterioration of daily living activities and quality of life. In our study, EQ-5D index was significantly higher in the KP group than that in the VP group. More restoration of vertebral height and kyphosis correction may have resulted in better quality of life in the aspect of mobility, pain, and anxiety or depression.

The main limitation of our study is that we compared quality of life between the VP group and the KP group without measuring quality of life before at preoperative status. As a result, we could not calculate the improvement of quality of life after the procedures compared to the preoperative status. In addition, misclassification of the medical condition might be possible, which might have resulted in an underestimation of their effect on the quality of life. Furthermore, clinical outcome was based on VAS and EQ-5D dimensions in this study. There are other measures for quality of life, such as 36-Item Short Form Health Survey (SF-36), which should have been taken into account to make this study more valuable.

\section{Conclusions}

Health related quality of life in osteoporotic vertebral fracture patients who received balloon KP was significantly better than that of patients who underwent VP in the aspect of mobility, pain, and anxiety or depression. In addition, pain was the most impaired dimension after both procedures. Therefore, balloon KP should be considered as a better choice than VP to achieve a better quality of life in patients with OVFs.

\section{Conflict of Interest}

No potential conflict of interest relevant to this article was reported.

\section{References}

1. Cockerill W, Lunt M, Silman AJ, et al. Health-related quality of life and radiographic vertebral fracture. Osteoporos Int 2004;15:113-9.

2. de Oliveira PP, Sandrin CC, Batista PH, Marinheiro LP, Wender MC, Roisenberg F. Relationship between quality of life and vertebral fracture in older women living in Southern Brazil. Rev Bras Ginecol Obstet 2011;33:231-7. 
3. Hall SE, Criddle RA, Comito TL, Prince RL. A casecontrol study of quality of life and functional impairment in women with long-standing vertebral osteoporotic fracture. Osteoporos Int 1999;9:508-15.

4. Naves Diaz M, Diaz Lopez JB, Rodriguez Rebollar A, Gomez Alonso C, Diaz Corte C, Cannata Andia J. Effect of vertebral fracture on health related quality of life in a Spanish population older than 54 years. Med Clin (Barc) 2001;116:533-5.

5. Dickman CA, Fessler RG, MacMillan M, Haid RW. Transpedicular screw-rod fixation of the lumbar spine: operative technique and outcome in 104 cases. J Neurosurg 1992;77:860-70.

6. Klazen CA, Lohle PN, de Vries J, et al. Vertebroplasty versus conservative treatment in acute osteoporotic vertebral compression fractures (Vertos II): an openlabel randomised trial. Lancet 2010;376:1085-92.

7. Wardlaw D, Cummings SR, Van Meirhaeghe J, et al. Efficacy and safety of balloon kyphoplasty compared with non-surgical care for vertebral compression fracture (FREE): a randomised controlled trial. Lancet 2009;373:1016-24.

8. Yang DH, Cho KH, Chung YS, Kim YR. Effect of vertebroplasty with bone filler device and comparison with balloon kyphoplasty. Eur Spine J 2014;23:271825.

9. Li Z, Ni C, Chen L, et al. Kyphoplasty versus vertebroplasty for the treatment of malignant vertebral compression fractures caused by metastases: a retrospective study. Chin Med J (Engl) 2014;127:1493-6.

10. Stevenson M, Gomersall T, Lloyd Jones M, et al. Percutaneous vertebroplasty and percutaneous balloon kyphoplasty for the treatment of osteoporotic vertebral fractures: a systematic review and cost-effectiveness analysis. Health Technol Assess 2014;18:1-290.

11. Kong LD, Wang P, Wang LF, Shen Y, Shang ZK, Meng LC. Comparison of vertebroplasty and kyphoplasty in the treatment of osteoporotic vertebral compression fractures with intravertebral clefts. Eur J Orthop
Surg Traumatol 2014;24 Suppl 1:S201-8.

12. Albers SL, Latchaw RE. The effects of randomized controlled trials on vertebroplasty and kyphoplasty: a square PEG in a round hole. Pain Physician 2013;16: E331-48.

13. Diamond TH, Clark WA. Percutaneous vertebroplasty: a novel treatment for acute vertebral fractures. Med J Aust 2001;174:398-400.

14. Garfin SR, Yuan HA, Reiley MA. New technologies in spine: kyphoplasty and vertebroplasty for the treatment of painful osteoporotic compression fractures. Spine (Phila Pa 1976) 2001;26:1511-5.

15. Kim KH, Kuh SU, Chin DK, et al. Kyphoplasty versus vertebroplasty: restoration of vertebral body height and correction of kyphotic deformity with special attention to the shape of the fractured vertebrae. J Spinal Disord Tech 2012;25:338-44.

16. Sullivan PW, Lawrence WF, Ghushchyan V. A national catalog of preference-based scores for chronic conditions in the United States. Med Care 2005;43:736-49.

17. Wenger M, Markwalder TM. Surgically controlled, transpedicular methyl methacrylate vertebroplasty with fluoroscopic guidance. Acta Neurochir (Wien) 1999;141:625-31.

18. Hiwatashi A, Sidhu R, Lee RK, deGuzman RR, Piekut DT, Westesson PL. Kyphoplasty versus vertebroplasty to increase vertebral body height: a cadaveric study. Radiology 2005;237:1115-9.

19. Rollinghoff M, Siewe J, Zarghooni K, et al. Effectiveness, security and height restoration on fresh compression fractures--a comparative prospective study of vertebroplasty and kyphoplasty. Minim Invasive Neurosurg 2009;52:233-7.

20. Suzuki N, Ogikubo O, Hansson T. The prognosis for pain, disability, activities of daily living and quality of life after an acute osteoporotic vertebral body fracture: its relation to fracture level, type of fracture and grade of fracture deformation. Eur Spine J 2009;18:77-88. 